\title{
Structure and phase behavior of a two-dimensional system with core-softened and long-range repulsive interactions
}

\author{
Philip J. Camp* \\ School of Chemistry, University of Edinburgh, West Mains Road, Edinburgh EH9 3JJ, United Kingdom
}

(Received 4 August 2003; published 18 December 2003)

\begin{abstract}
The structure and phase behavior of a two-dimensional system with purely repulsive core-softened and long-range interactions are studied using Monte Carlo computer simulations. The pair interactions are of the form, $u(r)=4 \epsilon\left[(\sigma / r)^{12}-(\sigma / r)^{6}\right]+\epsilon^{\prime}(\sigma / r)^{3}$, with the energy parameter, $\epsilon^{\prime}=(8 \sqrt{6} / 9) \epsilon$ chosen to give a stationary point of inflection in the pair potential at $r=6{ }^{1 / 6} \sigma$. This potential approximates the effective interparticle interactions for a two-dimensional dipolar system in a strong field aligned perpendicular to the plane. The low-temperature portion of the phase diagram is sketched out, and the static properties of the various phases are analyzed in some detail. At low temperatures a variety of interesting states are in evidence, including: fluids with chainlike, striped, and 6-10 sided polygon structural motifs; low-density and highdensity triangular crystalline phases; and defective Kagomé lattices. It is shown that clustering is driven by the presence of the repulsive shoulder in the pair potential. Other features, such as the presence of a disordered phase with a network structure, are due to the long-range $\left(1 / r^{3}\right)$ repulsive tail in the potential. The relevance of the simulation results to experimental work, including materials synthesis, is briefly discussed.
\end{abstract}

DOI: 10.1103/PhysRevE.68.061506

PACS number(s): 64.70.Dv, 61.20.Ja, 61.20.Qg

\section{INTRODUCTION}

The theoretical description of the structure, properties, and dynamics of strongly interacting dipolar fluids remains a challenging topic in condensed-matter physics. The most common examples of such systems are colloidal ferrofluids, which are suspensions of roughly spherical, homogeneously magnetized particles of diameter $10 \mathrm{~nm}-10 \mu \mathrm{m}$, dispersed in a simple solvent. The magnetic particles are usually coated in a thin layer of inert nonmagnetic material to prevent irreversible aggregation. The effective interaction between a pair of particles is a sum of the anisotropic dipole-dipole interaction, plus a short-range interaction, $u_{\mathrm{SR}}(r)$, arising from the hard core and (usually weak) dispersion interactions. The pair potential can therefore be represented by

$$
u(r)=u_{\mathrm{SR}}(r)+\frac{\left(\boldsymbol{\mu}_{1} \cdot \boldsymbol{\mu}_{2}\right)}{r^{3}}-\frac{3\left(\boldsymbol{\mu}_{1} \cdot \mathbf{r}\right)\left(\boldsymbol{\mu}_{2} \cdot \mathbf{r}\right)}{r^{5}},
$$

where $\boldsymbol{\mu}_{i}$ is the dipole on particle $i, \mathbf{r}$ is the interparticle separation, and $r=|\mathbf{r}|$.

Early experimental studies of thin films of colloidal ferrofluids at low density and in the absence of a magnetic field, showed that strong but reversible clustering can arise whereby the dipoles line up "nose-to-tail" to form extended chainlike structures [1]. Very recent experiments using cryogenic techniques and electron microscopy have produced images of chaining and aggregation in ferrofluid films $[2,3]$. The aggregation of the particles to form chains is due almost entirely to the anisotropic dipole-dipole interaction, and in particular, to the minimum energy conformation of a pair of dipoles. Recently, Weis has studied the rich variety of structures adopted by such quasi-two-dimensional fluids using

*Email address: philip.camp@ed.ac.uk
Monte Carlo (MC) computer simulations [4,5]. In the absence of an applied magnetic field, the dipoles lie preferentially in the plane of the fluid, with very small fluctuations in the normal direction. At low temperatures, the dipoles are seen to align nose-to-tail, resulting in a high degree of chaining that persists throughout the entire density range. Even at high density, vortex and linear orientations of the dipoles are superimposed on the close-packed triangular positional lattice dictated by the short-range repulsions.

In recent experiments, it has been shown that bulk aqueous ferrofluids in applied magnetic fields can form a variety of field-induced quasi-two dimensional structures [6]. In these experiments, the colloidal particles align along the applied magnetic field to produce bundles of needles. These needles, when imaged along a direction parallel to the magnetic field, produce a disordered glassy phase at low field, and a "disconnected labyrinthine" or "striped-liquid" phase [7] at high field. This striped liquid is distinct from a true lamellar (striped) phase, in that there is no long-range ordering of layers (see Fig. 5 of Ref. [6]). A quasi-twodimensional lamellar phase was observed, however, when latex spheres were added to the ferrofluid suspension.

These studies illustrate how complex structures and phases can arise from quite simple effective interactions. In the same vein, in this work we study the structure and phase behavior of a model system which approximates a twodimensional system of dipolar particles in a strong field aligned perpendicular to the plane. The effective pair interaction potential is

$$
u(r)=4 \epsilon\left[\left(\frac{\sigma}{r}\right)^{12}-\left(\frac{\sigma}{r}\right)^{6}\right]+\epsilon^{\prime}\left(\frac{\sigma}{r}\right)^{3},
$$

where the first term is an assumed Lennard-Jones form for the nondipolar interaction, $\epsilon$ and $\sigma$ are the Lennard-Jones energy and distance parameters, respectively, and $\epsilon^{\prime}$ is an additional energy parameter. With the dipoles aligned normal 


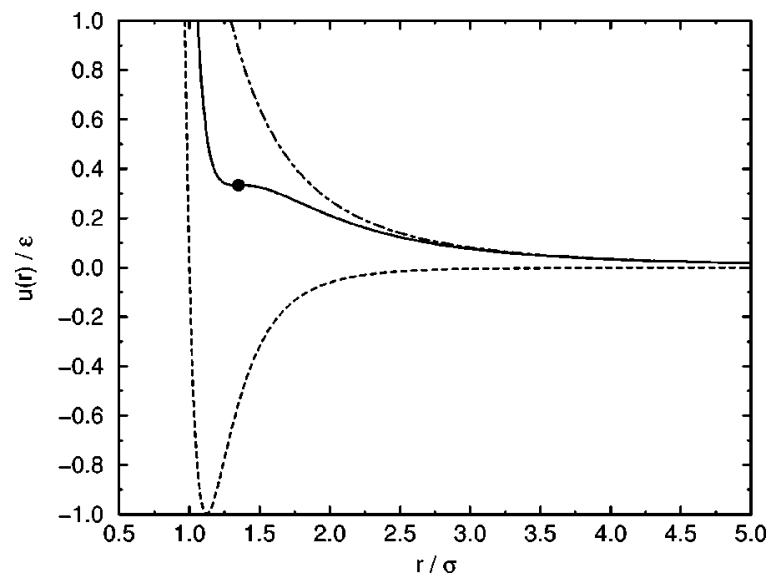

FIG. 1. Interaction potentials defined in Eqs. (2) and (3): Lennard-Jones interaction interaction potential (dashed line); repulsive potential $\epsilon^{\prime}(\sigma / r)^{3}$ with $\epsilon^{\prime} / \epsilon=8 \sqrt{6} / 9$ (dot-dashed line); total interaction potential $u(r)$ (solid line). The stationary point-ofinflection is marked by a filled circle.

to the plane of the fluid, the dipole part of the interparticle potential in Eq. (1) reduces to $\mu^{2} / r^{3}$ (purely repulsive), and hence can be identified with $\epsilon^{\prime}$ in Eq. (2). In this study, the value of $\epsilon^{\prime} / \epsilon$ is chosen to give a purely repulsive pair potential with no local minimum, and a stationary point of inflection (see Fig. 1):

$$
\begin{gathered}
\frac{\epsilon^{\prime}}{\epsilon}=\frac{8 \sqrt{6}}{9}=2.1773 \ldots, \\
\frac{r^{*}}{\sigma}=6^{1 / 6}=1.3480 \ldots, \\
\frac{u\left(r^{*}\right)}{\epsilon}=\frac{1}{3} .
\end{gathered}
$$

The asterisks (*) in Eqs. (4) and (5) denote values at the stationary point of inflection in $u(r)$. The pair potential is plotted in Fig. 1, along with the constituent Lennard-Jones and $1 / r^{3}$ repulsive interactions. It should be noted that in many ferrofluids, the attractive dispersion interactions are very weak compared to the dipole-dipole coupling, and hence such systems can be modeled as assemblies of dipolar hard spheres. By contrast, the energy parameter in Eq. (3) implies quite a large dispersion interaction compared to the dipole-dipole coupling.

The interaction potential specified in Eqs. (2) and (3) consists of a short-range "hard" core, a repulsive shoulder, and a long-range repulsive tail. There is considerable interest in the properties of systems with such "core-softened" potentials [8-16]. Recent activity in this area has been prompted largely by the discovery of density anomalies (expansion upon cooling at constant pressure) in network-forming fluids such as water [17], phosphorus [18], and carbon [19]. To explain these anomalies, it has been proposed that there exist second critical points in the phase diagrams. In the case of water, experimental and simulation results are consistent with there being a first-order low-density liquid to high- density liquid phase transition, terminating in a second critical point at lower temperature and higher pressure than the usual gas-liquid critical point (see Ref. [20] and references therein).

We note two earlier simulation studies on model potentials closely related to that defined in Eqs. (2) and (3). Reference [8] details ground-state calculations and finitetemperature simulations of a two-dimensional system with an interaction potential consisting of a hard core plus a linear repulsive ramp. Interestingly, with increasing density, a succession of unusual ground states is found, which includes chained, cubic, and honeycomb structures, in addition to the usual triangular close-packed lattice. A related model potential, consisting of a hard core plus a repulsive step, has recently been shown to give rise to a variety of unusual finitetemperature structures [16]. These include a low-density triangular solid lattice, disordered arrays of dimers and higher chainlike clusters, and apparently a lamellar (striped) structure [16]. In both of these model systems, clustering occurs to minimize the number of overlaps between neighboring particles.

In this work, the phase diagram of the two-dimensional model defined in Eqs. (2) and (3) is studied using MC computer simulations. It will be shown that the fluid and solid phases exhibit a variety of structural motifs arising from the presence of the repulsive shoulder and the long-range $1 / r^{3}$ repulsive tail in the pair potential. In particular, we find fluid phases exhibiting a high degree of clustering of the particles, resulting in dimerized, chainlike, and meshlike structures. The solid phases possess a variety of structures, depending on density, including low-density and high-density triangular lattices, and defective Kagomé structures. As well as being of fundamental interest, these results suggest a strategy for producing a range of self-assembled structures which could be exploited for use as templating or directing agents in materials syntheses.

This paper is organized as follows. In Sec. II, we summarize the simulation methods employed in the study. Sec. III contains the results of this work, pertaining to the phase behavior (III A), structure (III B), diffusion (III C), clustering (III D), chain formation (III E), and polygon formation (III F) in the model system. Sec. IV concludes the paper.

\section{SIMULATION DETAILS}

Canonical (NAT) MC simulations of $N$ particles were performed in square simulation cells of area $A=L^{2}$, and at temperature $T$; constant-tension constant-temperature (NPT) simulations were performed with tension, $P$ [21]. Periodic boundary conditions were applied in all cases. Unless stated otherwise, a system size of $N=500$ particles was employed; some simulations with $N=2000$ particles were also carried out to assess finite-size effects. Long-range corrections to thermodynamic properties were computed in the familiar fashion, by ignoring spatial correlations beyond $r=L / 2$ [21]. In the NAT simulations, one MC cycle consisted of, on average, one trial displacement per particle. In the NPT simulations, each MC cycle also included, on average, a trial displacement in $\ln A$, with the cell geometry fixed. In all 


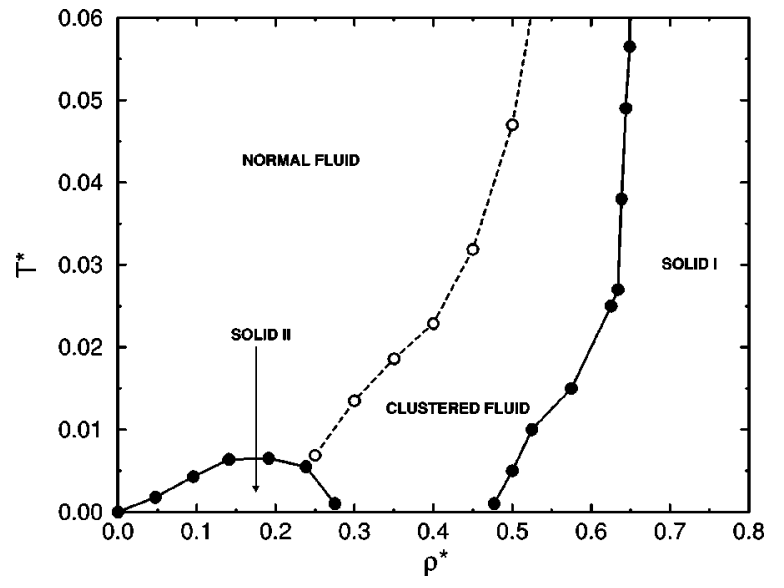

FIG. 2. Phase diagram in the density-temperature $\left(\rho^{*}-T^{*}\right)$ plane: phase boundaries assumed to be first order are represented by filled circles and solid lines; the crossover from high-temperature "normal" fluid to low-temperature "clustered" fluid is represented by open circles and the dashed line.

simulations, the maximum displacement parameters were adjusted to give $30 \%$ acceptance ratios. For the most part, NPT simulations were used to locate (apparently first-order) fluidsolid phase boundaries, while detailed calculations of structural properties were carried out during simulations in the NAT ensemble. The results of the simulations are reported in terms of the following reduced units: the reduced temperature $T^{*}=k_{\mathrm{B}} T / \epsilon$, where $k_{\mathrm{B}}$ is Boltzmann's constant; the reduced number density $\rho^{*}=N \sigma^{2} / A$; the reduced tension $P^{*}$ $=P \sigma^{2} / \epsilon$; the reduced configurational energy $U^{*}=U / \epsilon$; the reduced enthalpy $H^{*}=(U+P A) / \epsilon$.

To help identify fluid phases, the diffusion of the particles was monitored by calculating the mean-squared displacement $\left\langle R^{2}(\tau)\right\rangle$ using the expression

$$
\left\langle R^{2}(\tau)\right\rangle=\frac{1}{N} \sum_{i=1}^{N}\left|\mathbf{r}_{i}(\tau)-\mathbf{r}_{i}(0)\right|^{2}
$$

where $\mathbf{r}_{i}(\tau)$ is the position of the $i$ th particle after $\tau$ MC cycles. In calculating this quantity, we allow the particles to leave the central simulation cell. Although there is no real dynamical information in MC simulations, $\left\langle R^{2}(\tau)\right\rangle$ should increase linearly in fluid phases, assuming a fixed MC acceptance ratio.

\section{RESULTS}

\section{A. Phase behavior}

In Fig. 2, we present the low-temperature portion of the phase diagram in the density-temperature $\left(\rho^{*}-T^{*}\right)$ plane, showing the fluid-solid phase boundaries. Note the presence of two regions of stability of solid phases. The fluid-solid phase boundaries were determined by NPT-MC simulations. It should be remembered that it is still not proven that twodimensional melting in hard-core systems proceeds as in the Kosterlitz-Thouless-Halperin-Nelson-Young (KTHNY) scenario [22-25], i.e., via an intermediate hexatic phase linked to the fluid and solid phases by continuous transitions. A

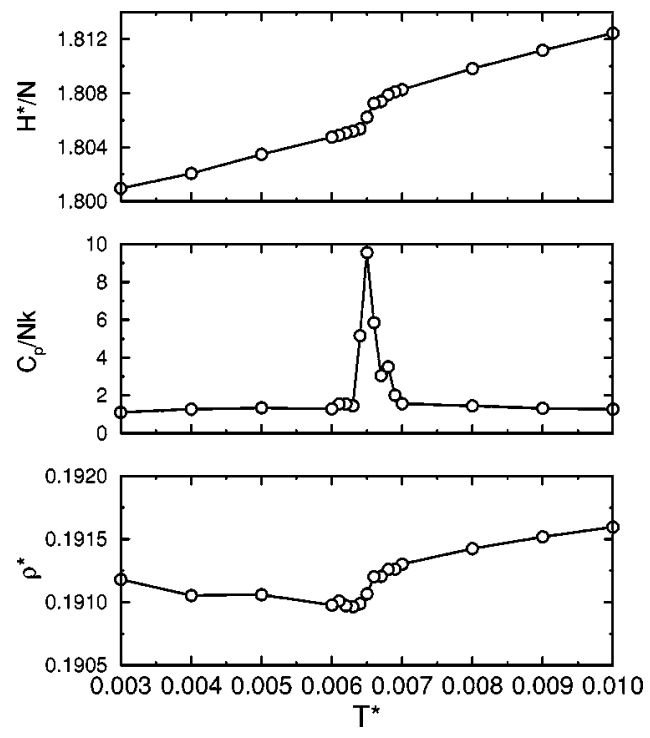

FIG. 3. Thermodynamic properties as functions of temperature along the isobar with $P^{*}=0.2$ : configurational enthalpy $H^{*} / N$ (top); configurational heat capacity $C_{p} / N k_{\mathrm{B}}$ (middle); density $\rho^{*}$ (bottom). The lines are guides to the eye.

recent simulation study of hard disks yielded evidence compatible with distinct fluid-hexatic and hexatic-solid transitions, but no direct observation of the hexatic phase was possible [26]. It is beyond the scope of this study to investigate the possibility of a KTHNY scenario with the current interaction potential, but it would be interesting to study the effects of the repulsive shoulder in the potential. With these points in mind, the fluid-solid phase boundaries have been determined by locating sharp features in the enthalpy, heat capacity, and density, as functions of temperature at fixed tension.

As an example, in Fig. 3 we show various thermodynamic properties computed along an isobar with $P^{*}=0.2$. On the scale of Fig. 2, in the temperature range $0.003 \leqslant T^{*} \leqslant 0.010$ this particular isobar is an almost vertical line with $\rho^{*}=0.19$. In Fig. 3, we see a sharp feature in the enthalpy $H^{*}$, and a peak in the heat capacity $C_{P}$ at a temperature of $T^{*}$ $=0.0065$. An inspection of simulation snapshots confirmed that the sharp features at $T^{*}=0.0065$ are due to a transition between a high-temperature fluid and a low-temperature triangular lattice; these phases will be illustrated in Sec. III B. Above $T^{*}=0.0065$, the density increases with increasing temperature, implying a negative thermal expansion coefficient $\alpha_{P}=V^{-1}(\partial V / \partial T)_{P}$. This is illustrated in more detail in Fig. 4, which shows the density as a function of temperature in the region $T^{*} \leqslant 0.020 ; \alpha_{P}>0$ in the regions $T^{*}<0.0065$ and $T^{*} \geq 0.015$, whereas $\alpha_{P}<0$ in the range $0.0065 \leqslant T^{*}$ $\lessgtr 0.015$. The magnitude of this anomaly is small; the density drops by less than $1 \%$ as the system is cooled from $T^{*}$ $=0.015$ to $T^{*}=0.0065$. It has been suggested that such anomalies arise in two dimensions due to the freezing transition being quasicontinuous, as in the KTHNY scenario, and so crystalline clusters can coexist with the fluid in finite-size simulations at constant chemical potential or pressure [14]. The explanation for the behavior in Fig. 4 is not clear, how- 


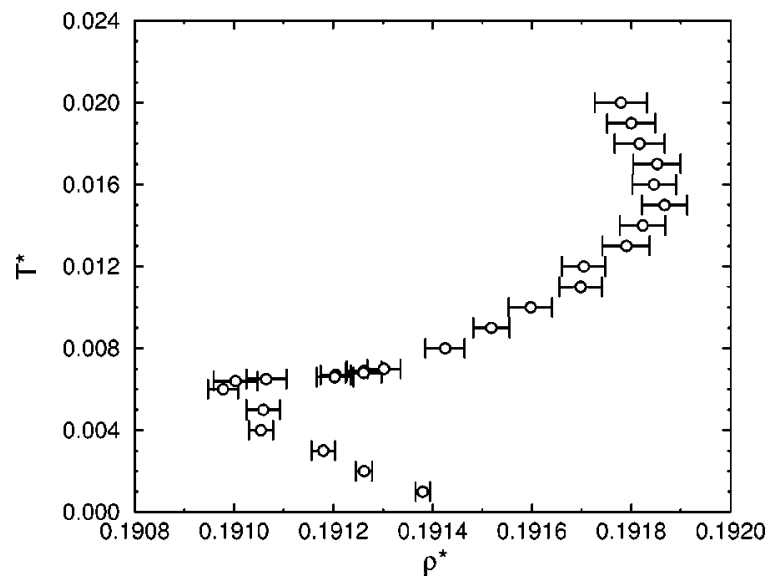

FIG. 4. Density $\rho^{*}$ as a function of temperature $T^{*}$ along the isobar with $P^{*}=0.2$, in the vicinity of the fluid-solid transition.

ever, since no such crystalline ordering is apparent in finitesize simulations above $T^{*}=0.0065$. In addition, no such anomalies were found for other isobars, although since the size of the effect is so small, it is possible that other state points with $\alpha_{P}<0$ were missed.

The fluid-solid boundaries were determined in a similar fashion along isobars in the range $8.0 \times 10^{-3} \leqslant P^{*} \leqslant 7.0$, although as is shown below, the structural characteristics of the fluid and solid phases change dramatically with increasing pressure. No attempts were made to resolve the fluid and solid coexistence densities, hence the phase boundaries are represented as single lines on Fig. 2. Note that at high temperature there is only one stable solid phase.

\section{B. Structure}

The structural features of the system at low temperatures are fascinating. As an illustration, in Fig. 5 we show snapshots from NAT-MC simulations spanning the density range $0.05 \leqslant \rho^{*} \leqslant 0.70$ along the isotherm $T^{*}=0.005$. Starting at $\rho^{*}=0.05$, the system is clearly in an isotropic fluid phase (labeled a normal fluid in Fig. 2), although the snapshot is superficially similar to the columnar glassy phase reported in Fig. 5 of Ref. [6]. At a density of $\rho^{*}=0.15$, the system is in the solid II region of the phase diagram; the snapshot clearly shows the low-density triangular lattice structure. Upon compression to $\rho^{*}=0.25$, the system moves out of the triangular solid II phase, and exhibits a largely dimerized structure, although some trimers are also in evidence. At $\rho^{*}=0.30$ the clustering has increased to such a degree that chainlike structures are apparent; the structure is reminiscent of the labyrinthine phase reported in Ref. [6]. At $\rho^{*}=0.35$ the chains are spanning the simulation cell, and the resulting structure is suggestive of a lamellar stripe phase; a similar structure has been reported in systems with step potentials at low temperatures and at comparable reduced densities (reported in units of the hard-core diameter) [16]. Chain formation and the possible existence of a lamellar phase are discussed in more detail in Sec. III E. At densities of $\rho^{*}=0.40$ and $\rho^{*}=0.45$, the structures appear to consist of tilings of closed polygons, with the polygon size decreasing with increasing density. Domains with the Kagomé lattice structure emerge at a den-
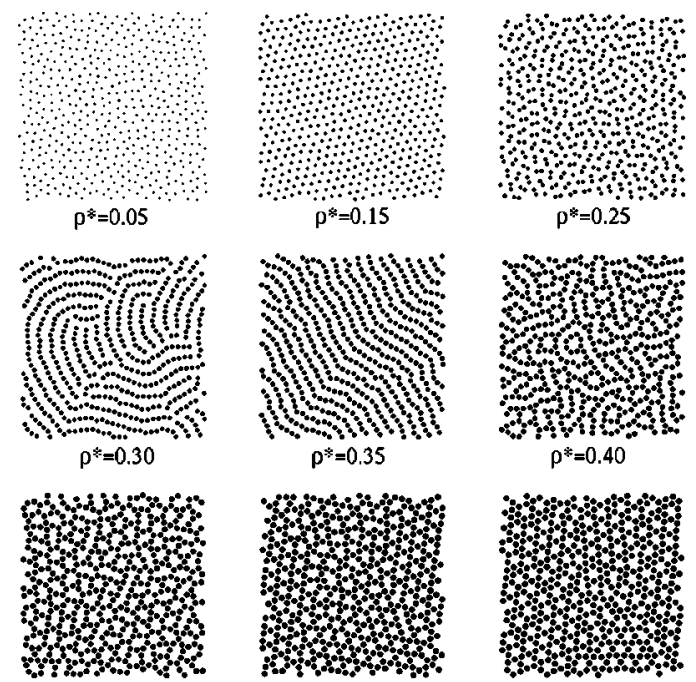

$\rho^{*}=0.45$

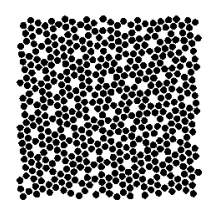

$\rho^{*}=0.50$

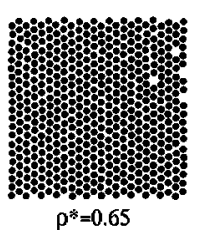

$\rho^{*}=0.55$

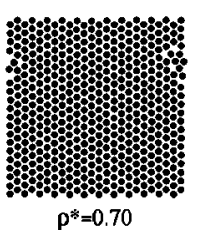

FIG. 5. Snapshots from $N A T$-MC simulations of $N=500$ particles along the isotherm with $T^{*}=0.005$. Particles are drawn with diameter $1 \sigma$.

sity of $\rho^{*}=0.50$, apparently in coexistence with a droplet of the fluid phase located near the center of the simulation cell. In the Kagomé structure, each lattice point is connected to four others. At progressively higher densities the vacancies gradually fill up, giving ultimately a close-packed triangular lattice.

Figure 5 shows that at low temperature and moderate densities, the particles prefer to cluster, rather than to retain as diffuse a structure as possible. To explore this effect in more detail, we computed the radial distribution function (rdf) $g(r)$; some representative results for the isotherm with $T^{*}$ $=0.005$ are shown in Fig. 6. At a density of $\rho^{*}=0.05$, the system is in the normal fluid phase, which clearly shows no long-range ordering. From the position of the first peak in the rdf, one can estimate the effective hard-core diameter to be $d \simeq 4.5 \sigma$, which is comparable to the mean separation $1 / \sqrt{\rho}$ $=4.47 \sigma$. The system is in the solid II phase at $\rho^{*}=0.15$, and the triangular lattice structure is reflected in the rdf; the effective hard-core diameter is estimated to be $d \simeq 2.7 \sigma$, again close to the mean separation $1 / \sqrt{\rho}=2.58 \sigma$. At a density of $\rho^{*}=0.25$, the system is largely dimerized (see Fig. 5), and the clustering is reflected in a narrow peak in the rdf centered at $r \simeq 1.25 \sigma$. This is significantly smaller than the mean separation $1 / \sqrt{\rho}=2.00 \sigma$ and corresponds to the region of the narrow plateau in the interaction potential (see Fig. 1). Therefore, it is the repulsive shoulder in $u(r)$ that is responsible for the apparent clustering, or "core collapse" [8], of the particles. Before going on to discuss clustering in more detail, the following section deals with the fluid nature of the clustered states. 


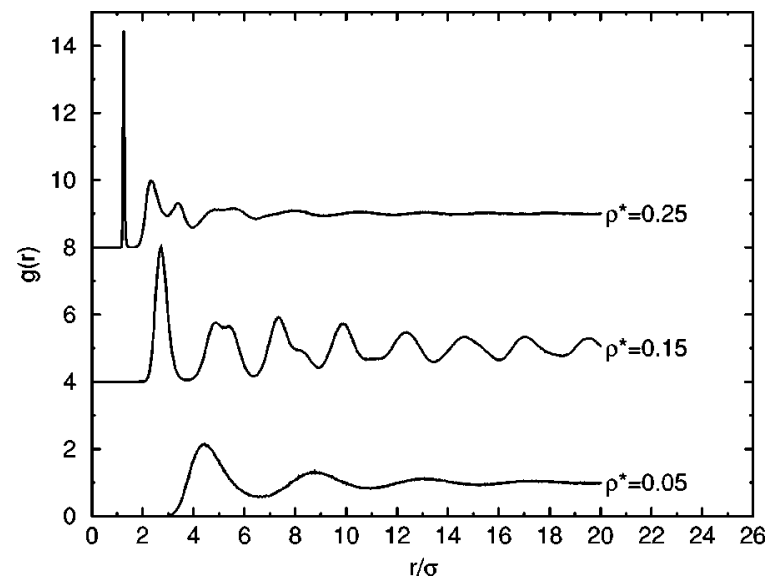

FIG. 6. Radial distribution function $g(r)$ at three densities along the isotherm with $T^{*}=0.005: \rho^{*}=0.05$ (bottom), $\rho^{*}=0.15$ (middle), $\rho^{*}=0.25$ (top). The curves are displaced by four units for clarity.

\section{Diffusion}

The mean-squared displacement, Eq. (6), is shown for three densities along the isotherm with $T^{*}=0.005$, in Fig. 7 . At a density of $\rho^{*}=0.25$, the "diffusion" clearly indicates a fluid phase. At $\rho^{*}=0.35$ and $\rho^{*}=0.45$, however, the curves appear almost flat; on an expanded scale the curves still rise roughly linearly, but clearly the dynamics at these state points are characterized by very slow single-particle motions. The simulation snapshots in Fig. 5 suggest that the dynamics at this temperature, and in the density range $0.30 \leqslant \rho^{*} \leqslant 0.45$, should be dominated by very slow collective motions, and it is even possible that these states should best be classified as amorphous, or glassy. It would be very interesting to investigate the dynamics in this system, and molecular dynamics (MD) simulations of this system are in progress. In the meantime, these manifestly noncrystalline states will be referred to as "fluids," with the proviso that subsequent dynamical studies may show them to be glassy.

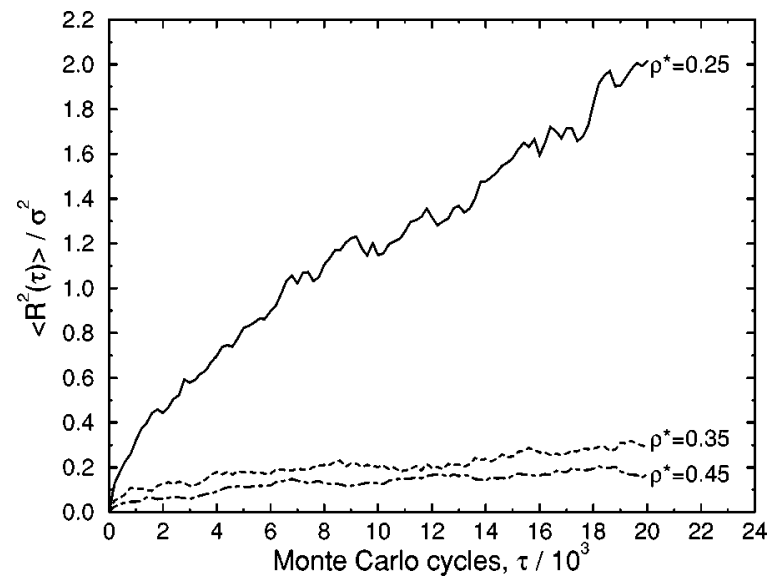

FIG. 7. Mean-squared displacement $\left\langle R^{2}(\tau)\right\rangle$ as a function of Monte Carlo cycle at three densities along the isotherm with $T^{*}$ $=0.005: \rho^{*}=0.25$ (solid line); $\rho^{*}=0.35$ (dashed line); $\rho^{*}=0.45$ (dot-dashed line).

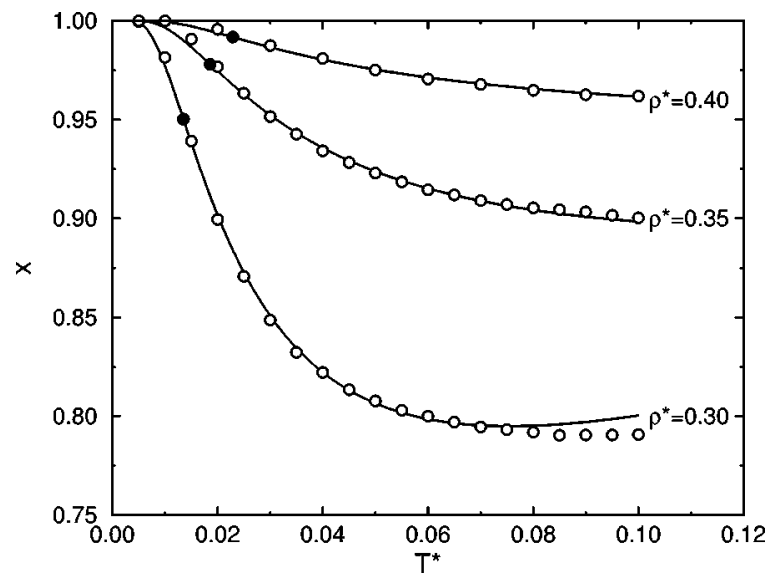

FIG. 8. Fraction of aggregated particles, $x$, as functions of temperature $T^{*}$ along three isochores, $\rho^{*}=0.30$ (bottom), $\rho^{*}=0.35$ (middle), and $\rho^{*}=0.40$ (top): simulation results (open circles); fits from Eq. (8) (solid lines); crossover temperatures (filled circles).

\section{Clustering}

Clustering is the result of a balance between energy and entropy (packing), but roughly speaking it arises from a requirement to minimize the number of overlaps between the repulsive shoulders on the particles. The clustering of the particles can be quantified with the help of a distance-based criterion. The rdf at $\rho^{*}=0.25$ in Fig. 6 shows that the cluster peak does not extend beyond about $1.5 \sigma$, referring to Fig. 1, this distance corresponds quite closely to the point at which the potential begins to die away. Hence, in the following we shall designate a pair of particles separated by less than $1.5 \sigma$ as belonging to the same cluster.

To examine the degree of aggregation as a function of temperature, we computed the fraction of particles that have at least one neighbor within $1.5 \sigma$, denoted by $x$. This quantity is plotted as a function of temperature along three isochores with $\rho^{*}=0.30,0.35$, and 0.40 , in Fig. 8; the curves for higher densities exhibit smaller deviations from $x=1$. We have analyzed the low-temperature variation of $x$ using a simple quasichemical equilibrium model. At very low temperatures, almost all of the particles are incorporated in clusters. As the temperature is raised, some particles will 'break free' of the clusters, accompanied by changes in the energy and entropy. Assuming that the concentration of "free" particles is small, and hence that these particles do not interact appreciably with one another, we approximate the free energy $F$ by

$$
\begin{aligned}
\frac{F}{N}= & k_{\mathrm{B}} T\left[\ln \rho \Lambda^{3}-1+x \ln x+(1-x) \ln (1-x)\right] \\
& +(1-x)\left(\Delta u_{0}-T \Delta s_{0}+C T^{2}\right),
\end{aligned}
$$

where the first term in square brackets is the ideal-gas contribution, $\Lambda$ is the de Broglie thermal wavelength of the particles, $\Delta u_{0}$ and $\Delta s_{0}$ are the energy and entropy costs, respectively, of turning a clustered particle into a free particle (by creating an exclusion zone of radius $1.5 \sigma$ ) when there are no other free particles in the system (i.e., at very low tempera- 


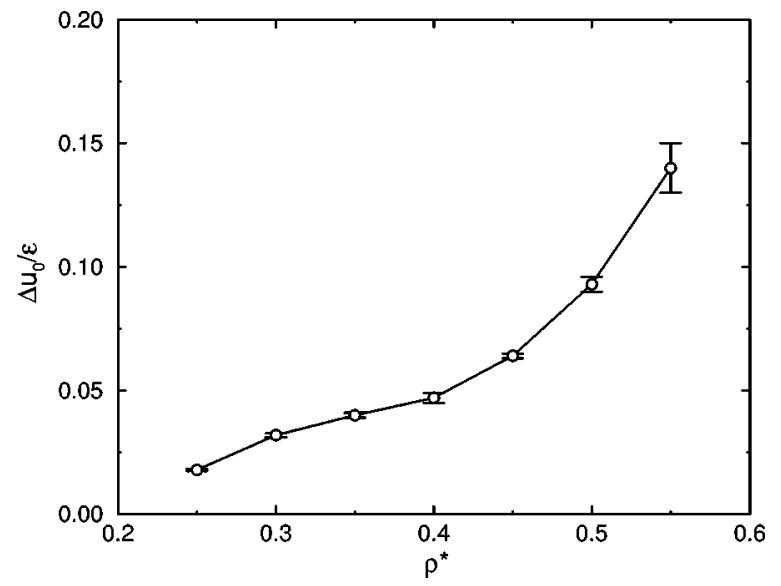

FIG. 9. Density dependence of the energy change $\Delta u_{0}$ associated with creating an exclusion zone of radius $1.5 \sigma$ around an initially aggregated particle.

ture), and $C$ is related to a higher thermodynamic derivative of $F$. Minimizing the free energy with respect to $x$ yields,

$$
\begin{gathered}
x \simeq \frac{Q(T)}{1+Q(T)}, \\
Q(T)=\exp \left(\frac{\Delta u_{0}}{k_{\mathrm{B}} T}-\frac{\Delta s_{0}}{k_{\mathrm{B}}}+\frac{C T}{k_{\mathrm{B}}}\right) .
\end{gathered}
$$

These expressions are only going to be valid at low temperature, and when the fraction of free particles is small. Equations (8) and (9) were fitted to simulation results along isochores with densities in the range $0.25 \leqslant \rho^{*} \leqslant 0.55$. The fits for $\rho^{*}=0.30,0.35$, and 0.40 are included in Fig. 8. The approximate expressions presented here are merely adequate to fit the simulation results at low density, largely because the fraction of free particles is significant, but the quality of the fit does improve with increasing density. The fit parameters $\Delta u_{0}$ and $\Delta s_{0}$ are plotted as functions of density in Figs. 9 and 10, respectively. Figure 9 shows the energy change $\Delta u_{0}$ increasing with density, due mainly to the decreasing mean

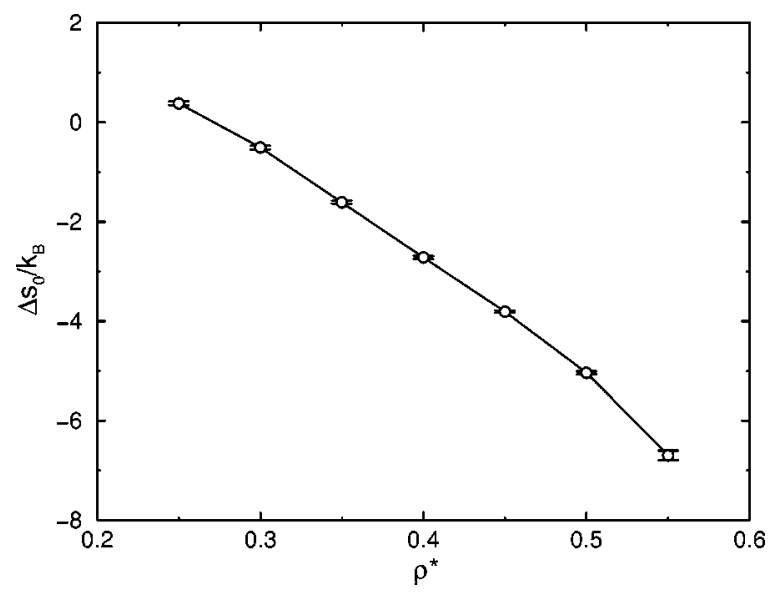

FIG. 10. Density dependence of the entropy change $\Delta s_{0}$ associated with creating an exclusion zone of radius $1.5 \sigma$ around an initially aggregated particle.

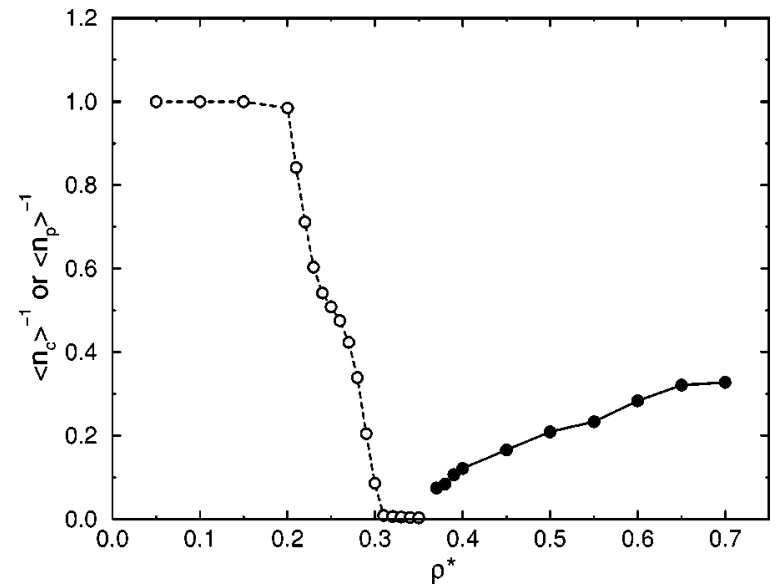

FIG. 11. Reciprocals of the average chain length $\left\langle n_{c}\right\rangle$ (open circles), and average polygon size $\left\langle n_{p}\right\rangle$ (filled circles), as functions of density along the isotherm with $T^{*}=0.005$. The lines are guides to the eye.

separation, and hence greater repulsion, between a free particle and its near neighbors (albeit at least $1.5 \sigma$ away). The associated entropy change $\Delta s_{0}$ decreases with increasing density, which reflects the decrease in free volume of a particle with an exclusion zone of radius $1.5 \sigma$.

Note that the high-temperature limit of $x$ increases with increasing density and decreasing mean separation, simply because the criterion for clustering is distance based. For each density, the temperature of maximum slope in $x$ was identified as a "crossover" temperature, roughly indicating the point at which the fully aggregated system begins to unbind appreciably. These temperatures for $\rho^{*}=0.30,0.35$, and 0.40 are indicated in Fig. 8. The locus of crossover temperatures over a wide density range is also plotted on the phase diagram in Fig. 2, and can be interpreted as delineating the boundary between the high-temperature normal fluid and the low-temperature clustered fluid. Having discussed the more general aspects of clustering in this system, we now move on to the specific formation of chains and polygons.

\section{E. Chaining}

In Ref. [16], a lamellar "striped" phase was observed in simulations of two-dimensional hard disks with a repulsive step potential extending out to one-and-a-half particle diameters. Similar structures are apparent in the present calculations in the density range $0.30 \leqslant \rho^{*} \leqslant 0.35$; see the snapshot at $\rho^{*}=0.35$ in Fig. 5. To examine chaining in more detail, we have computed the number of spheres per chain $n_{c}$ using the distance based criterion introduced in Sec. III D. In Fig. 11 we plot the reciprocal of the average of $n_{c}$ as a function of density along the isotherm with $T^{*}=0.005$. At $\rho^{*} \leqslant 0.2$, $\left\langle n_{c}\right\rangle^{-1} \simeq 1$, because there is no clustering. $\left\langle n_{c}\right\rangle^{-1}$ decreases sharply at a density of $\rho^{*}=0.2$, which corresponds to the approximate solid-fluid transition density at $T^{*}=0.005$. There appears to be a point of inflection at $\rho^{*}=0.25$, where $\left\langle n_{c}\right\rangle^{-1} \simeq 0.5$ corresponding to the dimerized state apparent in the simulation snapshots (see Fig. 5). The average chain length rises rapidly until a density of $\rho^{*}=0.31$, above which $\left\langle n_{c}\right\rangle^{-1}$ is effectively zero. 


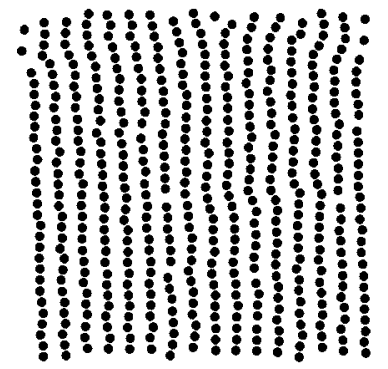

$N=500$

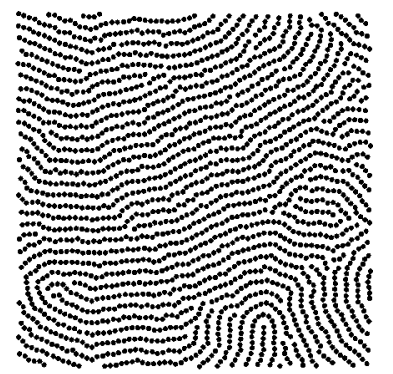

$\mathrm{N}=2000$
FIG. 12. Snapshots from $N A T$-MC simulations of $N=500$ particles (left) and $N=2000$ particles (right), at temperature $T^{*}$ $=0.005$ and density $\rho^{*}=0.31$. Particles are drawn with diameter $1 \sigma$.

In the $N=500$ particle system at $T^{*}=0.005$ and $\rho^{*}=0.31$, an almost perfect lamellar structure is formed; a snapshot from a simulation under these conditions is shown in Fig. 12. To check for finite-size effects, a simulation with $N=2000$ particles was performed at the same temperature and density; a snapshot is included in Fig. 12. It is clear that the perfect lamellar structure in the $N=500$ particle system is artificially stabilized by the periodic boundary conditions; effectively, the chainlike clusters extend over the entire length of the simulation cell, and join up with their periodic images. By contrast, there are many defects in the $N=2000$ system, largely consisting of disclinations. This indicates that there is likely no stable lamellar phase in this system; indeed, the snapshot for the $N=2000$ particle system is more reminiscent of a labyrinthine or "striped liquid" phase [6,7], in that there is no long-range ordered layer structure.

To examine the onset of stripe formation further, we have computed $\left\langle n_{c}\right\rangle$ as a function of temperature at a fixed density of $\rho^{*}=0.31$. The results for both $N=500$ and $N=2000$ particle systems are shown in Fig. 13. Above $T^{*}=0.012$, the results are essentially independent of system size. Below this temperature, however, $\left\langle n_{c}\right\rangle^{-1}$ drops faster in the smaller system, reflecting the artificial stabilization of system-spanning

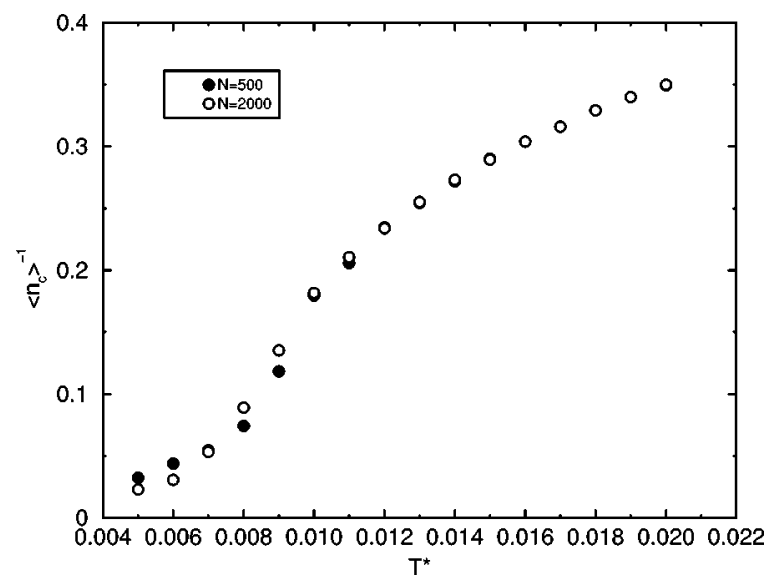

FIG. 13. Reciprocal of the average chain length $\left\langle n_{c}\right\rangle$ as a function of temperature along the isochore with $\rho^{*}=0.31$ : results from $N A T$-MC simulations of $N=500$ particles (filled circles); results from $N A T$-MC simulations of $N=2000$ particles (open circles).

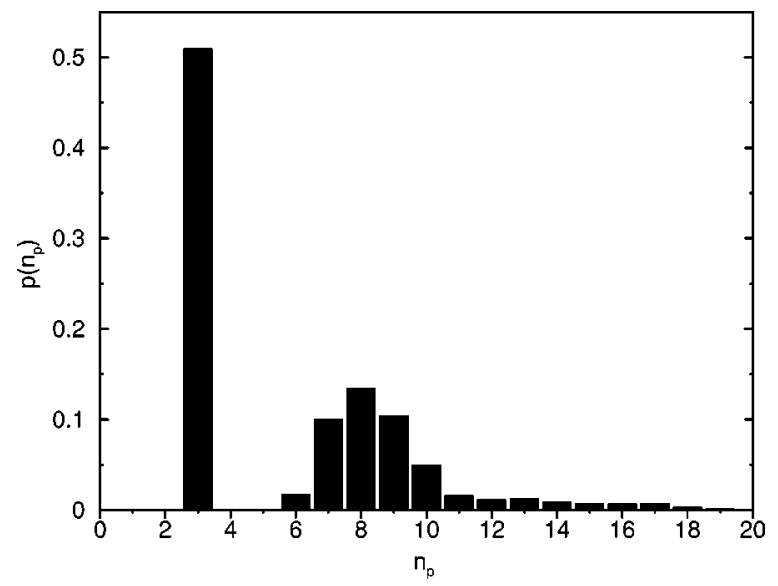

FIG. 14. Probability distribution function $p\left(n_{p}\right)$ of the number of particles per polygon $n_{p}$ at $\rho^{*}=0.45$ and $T^{*}=0.005$.

clusters afforded by the periodic boundary conditions. The results at low temperature correctly show that the larger system can accommodate larger chains. In quasi-twodimensional dipolar fluids, the formation of chains is not accompanied by discontinuities in any of the thermodynamic functions, and hence there is no "chaining" phase transition [27]. By analogy, it is unlikely that there is a phase transition in this system. This issue could be explored, however, by calculating the Binder cumulant $\left\langle\Phi^{2}\right\rangle^{2} /\left\langle\Phi^{4}\right\rangle$ for an order parameter $\Phi$ related to $n_{c}$. If there is a chaining phase transition, then the Binder ratios for different system sizes will intercept at the critical temperature. It is computationally demanding to achieve sufficiently good statistics for such finite-size scaling procedures, and is beyond the scope of this exploratory study.

\section{F. Polygon formation}

We now focus on the unusual low-temperature network structure made up of closed polygons, typified by the structure at $\rho^{*}=0.45$ and $T^{*}=0.005$ shown in Fig. 5. Using the distance-based criterion introduced in Sec. III D, we have calculated the number of particles in a given polygon, $n_{p}$. The reciprocal of the average of $n_{p}$ is plotted as a function of density at a temperature of $T^{*}=0.005$ in Fig. 11. At $\rho^{*}=0.35$ the structure is made up of chainlike clusters, and hence there are no polygons. Upon increasing the density, the chains form connections with neighboring chains, resulting in a tiling of the plane with polygons of decreasing size. As $\rho^{*}$ approaches the close-packed density, $\left\langle n_{p}\right\rangle^{-1} \rightarrow 1 / 3$, reflecting the onset of the triangular lattice structure.

The probability distribution function $p\left(n_{p}\right)$ is illuminating. This is shown at $\rho^{*}=0.45$ and $T^{*}=0.005$ in Fig. 14 . $49 \%$ of the polygons have four or more sides, and the average polygon size is 6.0 . The most probable polygon to be found (besides the triangle) is the octagon; this likely reflects the long-range repulsions between the particles, which keep opposite edges in the same polygon as far away from each other as possible, resulting in large (roughly circular) cavities forming in the system. 


\section{DISCUSSION}

The structure and phase behavior of a two-dimensional system with core-softened repulsive interactions have been studied using MC simulations. The essential features of the potential are a short-range hard-core repulsion, a repulsive shoulder containing a stationary point of inflection, and a long-range $1 / r^{3}$ repulsive tail.

The low-temperature portion of the phase diagram consists of low-density and high-density solids, separated by a phase with no long-range order. The low-density solid possesses a triangular lattice structure, whereas the high-density solid exhibits a succession of structures, including defective Kagomé, and close-packed triangular lattices. The lowtemperature fluid phase exhibits a variety of unusual structural motifs, including chains, stripes, and higher polygons such as octagons. The presence of clusters is a direct result of the particles minimizing the number of overlaps with neighboring particles; the formation of structures with higher polygons is also favored by the long-range $1 / r^{3}$ repulsions. The impact of these complex structures on the dynamical properties is likely to be significant; the results from MD simulations of the model system will be reported in a future paper.

With regard to the relevance of these results to experimental work, it is helpful to consider some order-of- magnitude estimates of the system parameters that would be required to produce the structures observed in the simulations at, say, $T^{*} \sim 10^{-2}$. At a temperature of $300 \mathrm{~K}$, the barrier height in the interaction potential would have to be $\sim 10^{4} \mathrm{~K}$ in units of $k_{\mathrm{B}}$; this represents a very strong interaction, which is unlikely to be achieved through dispersion and magnetic interactions. Other effective interactions, such as depletion forces, might prove more suitable for this purpose. With regard to suitable surface concentrations, for nanometer-sized particles, $\rho^{*} \sim 0.1$ corresponds to a real number density $\sim 10^{-3} \AA^{-2}$. The gains from being able to control the structures of thin films are manifold. Potential applications which immediately come to mind are the use of striped phases in the directed growth of molecular wires or carbon nanotubes, and the use of Kagomé structures as templating or pattering agents in the production of porous membranes. Despite the likely difficulty of realizing the model system in the laboratory, the results presented in this paper illustrate the complexity of structures than can arise from very simple isotropic interactions.

\section{ACKNOWLEDGMENT}

The support of the Engineering and Physical Sciences Research Council through the purchase of computer hardware under Grant No. GR/R45727/01 is gratefully acknowledged.
[1] P.H. Hess and P.H. Parker, Jr., J. Appl. Polymer Sci. 10, 1915 (1966)

[2] K. Butter, P.H.H. Bomans, P.M. Frederik, G.J. Vroege, and A.P. Philipse, Nat. Mater. 2, 88 (2003).

[3] K. Butter, P.H. Bomans, P.M. Frederik, G.J. Vroege, and A.P. Philipse, J. Phys.: Condens. Matter 15, S1451 (2003).

[4] J.J. Weis, Mol. Phys. 100, 579 (2002).

[5] J.J. Weis, J. Phys.: Condens. Matter 15, S1471 (2003).

[6] M.F. Islam, K.H. Lin, D. Lacoste, T.C. Lubensky, and A.G. Yodh, Phys. Rev. E 67, 021402 (2003).

[7] M. Seul and V.S. Chen, Phys. Rev. Lett. 70, 1658 (1993).

[8] E.A. Jagla, Phys. Rev. E 58, 1478 (1998).

[9] E.A. Jagla, J. Chem. Phys. 111, 8980 (1999).

[10] E.A. Jagla, Phys. Rev. E 63, 061501 (2001).

[11] E.A. Jagla, Phys. Rev. E 63, 061509 (2001).

[12] G. Malescio and G. Pellicane, Phys. Rev. E 63, 020501 (2001).

[13] G. Malescio, G. Franzese, G. Pellicane, A. Skibinsky, S.V. Buldyrev, and H.E. Stanley, J. Phys.: Condens. Matter 14, 2193 (2002).

[14] N.B. Wilding and J.E. Magee, Phys. Rev. E 66, 031509 (2002).

[15] G. Franzese, G. Malescio, A. Skibinsky, S.V. Buldyrev, and
H.E. Stanley, Phys. Rev. E 66, 051206 (2002).

[16] G. Malescio and G. Pellicane, Nat. Mater. 2, 97 (2003).

[17] P.H. Poole, F. Sciortino, U. Essmann, and H.E. Stanley, Nature (London) 360, 324 (1992).

[18] Y. Katayama, T. Mizutani, W. Utsumi, O. Shimomura, M. Yamakata, and K. Funakoshi, Nature (London) 403, 170 (2000).

[19] J.N. Glosli and F.H. Ree, Phys. Rev. Lett. 82, 4659 (1999).

[20] P. G. Debenedetti, Metastable Liquids: Concepts and Principles (Princeton University Press, Princeton, 1998).

[21] M. P. Allen and D. J. Tildesley, Computer Simulation of Liquids (Clarendon Press, Oxford, 1987).

[22] J.M. Kosterlitz and D.J. Thouless, J. Phys. C 6, 1181 (1973).

[23] B.I. Halperin and D.R. Nelson, Phys. Rev. Lett. 41, 121 (1978).

[24] D.R. Nelson and B.I. Halperin, Phys. Rev. B 19, 2457 (1979).

[25] A.P. Young, Phys. Rev. B 19, 1855 (1979).

[26] K. Binder, S. Sengupta, and P. Nielaba, J. Phys.: Condens. Matter 14, 2323 (2002).

[27] J.J. Weis, J.M. Tavares, and M.M. Telo da Gama, J. Phys.: Condens. Matter 14, 9171 (2002). 